\title{
Effects of different acquisition procedures on response variability
}

\author{
PATRICLA D. STOKES, FRANCIS MECHNER, and PETER D. BALSAM \\ Barnard College, Columbia University, New York, New York
}

\begin{abstract}
In three experiments with college students, the effects of different acquisition procedures on response variability were studied. The computer keypressing task involved learning a sequence with a minimum number of presses on a subset of the keyboard. Procedures differed in type of training and in the number, size, and sequence of training steps. Experiment 1 showed that instructions and shaping in three steps generated less variability in the number of responses made in each keypress sequence than shaping in six steps. Subsequent experiments showed that a large increase in the response requirement early in shaping increased variability. Postacquisition variability remained unchanged in the number of responses per sequence-the aspect of responding on which reinforcement was contingent-but declined in location and timing of keypressing. The results are discussed in terms of the implicit reinforcement of variability and how the acquisition of qualitatively different response strategies could influence variability.
\end{abstract}

Behavioral variability accompanies learning (Skinner, 1981; Staddon, 1993) and persists after learning has occurred (Anderson, 1993; Siegler, 1995). Evidence that variability accompanies learning comes from studies showing that it increases, temporarily, prior to the discovery of new problem-solving strategies. This happens when children learn to solve arithmetic problems (Siegler \& Jenkins, 1989), master grammatical rules in English (Bowerman, 1982), or acquire novel concepts (Goldin-Meadow, Alibani, \& Church, 1993). A similar phenomenon is seen in adults making novice-to-expert transitions in radiology (Lesgold et al., 1988) or cardiology (Johnson et al., 1981). Greater variability precedes acquisition of advanced diagnostic expertise. It has also been shown that greater variability during learning is associated with greater learning. Children who use more strategies at the start of training on a task acquire the correct strategy more often than those with fewer initial strategies (Siegler, 1995).

Once learning occurs, variability may decline but does not disappear. Its persistence is well documented in the problem-solving literature. In computer programming (Anderson, Conrad, \& Corbett, 1989), arithmetic (Carpenter \& Moser, 1982; Siegler \& Crowley, 1991), and causal attribution (Kuhn \& Phelps, 1982; Schauble, 1990), individuals use multiple solution paths, not only for the same sort of problem (say, addition), but for exactly the same problem. In these studies, variability results primarily from

This research was presented, in part, at a 1994 meeting of the Eastern Psychological Association in Providence, RI. It was supported by a grant from the Betty Lipsam foundation. The authors thank Joshu Goldberg, Darrin DuFord, and Douglas Madden for programming and Karen Zechowy for running subjects and analyzing data. Address correspondence to P. D. Stokes, Department of Psychology, Barnard College, Columbia University, New York, NY 10027-6598 (e-mail: pstokes@) barnard columbia.edu). switches between correct strategies, rather than from erroneous responding.

Finally, not only does variability persist after skill acquisition, it seems to persist at high levels in more accomplished individuals - at least in their domains of expertise. Intellectually gifted children and adults have been characterized as more curious, experimental, and explorative than those not in the gifted category (Janos \& Robinson, 1985), and renowned scientists and artists work on a variety of related projects (Wallace \& Gruber, 1989).

Given that there are consistent individual differences in variability, it is reasonable to ask, what accounts for them? One answer is that variability not only accompanies learning but is itself a learned aspect of behavior (Neuringer, 1993; Stokes, 1995b).

Support for the view that individuals learn how variably to behave comes from studies that reinforce current responses that are different from prior ones. Since reward is directly contingent on variability, this type of training can be said to involve explicit variability criteria. High variability levels can be shaped by rewarding novel responses (Goetz \& Baer, 1973; Holman, Goetz, \& Baer, 1977; Pyror, Haag, \& O'Reilly, 1969) or by rewarding less frequent, but not necessarily new, responses (Machado, 1994; Neuringer, 1992). The greater the difference required between current and prior responses, the higher the variability obtained. For example, variability is greater when sequences of right-left keypresses have to differ from 10 rather than from 5 previous sequences (Page \& Neuringer, 1985). Explicit instructions work as well. Telling students to do a task in different ways increases variability (Joyce \& Chase, 1990; Royce, 1898). In general, increases in variability resulting from explicit reinforcement contingencies are specific to a training domain (Baer, 1994). However, Eisenberger and Selbst (1994) report that rewarding highly divergent thinking in 
a word construction task increased originality on a subsequent drawing task.

Other work has focused on variability in tasks in which reinforcement is not explicitly contingent on how variably the task is done. In these studies, differences in acquisition procedures generate different levels of variation in the same task. One manipulation that affects variability involves placing different topographic contingencies on response form. Rats shaped to barpress under an initially high constraint (right paw only) were more variable than those shaped with a low initial constraint (press any way), even after the constraints were reversed (Stokes, 1995a).

Another factor that affects variability is the type of training procedure. Behavior is generally reported to be more variable when it is shaped than when it is instructed. However, with humans, shaping always includes some basic set of instructions. The basic set plus additional instructions are given to the groups designated as instructed. For example, in one study (Shimoff, Catania, \& Matthews, 1986), both shaped and instructed groups were told what points were worth and two ways in which they could be earned (pressing keys and guessing). The instructed groups were then told how to maximize earning points in keypressing or guessing. Given the above, it seems useful to place shaping and instruction on a continuum based on the degree of specificity in instruction (Joyce \& Chase, 1990). Excluding, of course, instructions to be variable or divergent, behavior is generally less variable when instructions about what responses to make are more completely specified. Variability levels generated by both types of training persist, despite changes in contingency (for a review, see Hackenberg \& Joker, 1994).

The overall goal of the present study was to more closely examine how variability is affected by differences in acquisition procedures. As mentioned previously, more variable performance is produced when shaping occurs with strong topographical constraints than with weaker constraints. The basis for this difference could be the number of shaping steps needed to acquire the response. Severe constraint (e.g., right-only barpressing) might produce more variable behavior because, de facto, it involves more shaping steps than does a less severe constraint (e.g., press any way). Similarly, shaping might produce more variable behavior than do instructions, because, in many studies, the shaping procedures involve more acquisition steps than do instructional procedures. Hence, the aspect of training that we initially focused on was the number of training steps.

An additional purpose of the present experiment was to examine variability in several aspects of responding. Earlier work has shown that variability is maintained in a targeted response dimension (Stokes, 1995a). However, we do not know what happens in other aspects of responding. Perhaps, like persistence (Eisenberger, 1992), variability is maintained only in the response dimension targeted by the explicit reward contingency. Alternatively, it may be maintained in other aspects as well. Thus, a secondary goal of the present study was to examine variabil- ity more broadly. To this end, the number, location, and timing of the target behavior were recorded and analyzed.

\section{EXPERIMENT 1 \\ Effects of Type of Training and Number of Training Steps}

Experiment 1 contrasted different training procedures and numbers of training steps to answer two questions. First, is variability a function of number of training steps? Second, are number of steps independent of whether training involves shaping or instruction? Type of training and number of steps can easily be confounded, because shaping usually involves several steps or approximations, whereas instructions can generate a target response in a single step. Thus, the subjects were shaped or instructed in either three or six steps. If number of steps is important, variability should be higher in the Six-Step group than in the Three-Step group. If type of training is important, variability might be higher in the shaped than in the instructed groups.

\section{Method}

\section{Subjects}

Forty undergraduates ( 29 female and 11 male) participated in the experiment in order to fulfill an introductory psychology class requirement.

\section{Apparatus and Stimuli}

Four personal computers, in separate $1.5 \times 3.5 \mathrm{~m}$ experimental rooms, were used to present stimuli and collect data. The door in each room was closed, to attenuate noise. The specific task was one adapted from previous work (Mechner, 1991; Mechner, Hyten, Field, \& Madden, 1997). As shown in the top panel of Figure 1, a mask was placed over each keyboard to make only the space bar, the Enter key, and eight alpha character keys (k l ; ' $\mathrm{m}, . /$ ) available for pressing. The character keys are presented in the figure and reported as ABCDEFGH.

The bottom panel of Figure 1 shows successive changes in the video display during the last phase of shaping, when the required press sequence started with the space bar, continued with at least 10 of character keypresses, and concluded with the Enter key. Prior to sequence initiation (A), a red rectangle appeared on a black background. When the space bar was pressed (B), the rectangle turned blue. When 10 character keypresses were made $(C)$, a white square appeared in the upper right corner of the blue rectangle. When the Enter key was pressed, the rectangle disappeared. If the sequence was correct (D), a point appeared on the screen; if it was incorrect, no point appeared. The display then reset to (A). If the space bar was not required, the display began with the blue rectangle (B). The white box $(C)$ only appeared during shaping. The subjects were not told that its appearance indicated that the required number of character presses was made.

\section{Procedure}

The target was the following press sequence; space bar, at least 10 presses on any combination of the eight character keys, Enter key.

The subjects were randomly assigned to one of four groups: Three- or Six-Step Shape, Three- or Six-Step Instruct. Group names refer to stages of training. There was one experimental session, with three phases separated by 5 -min breaks. Fifty points were earned for the target sequence during the acquisition phase, when the subjects were shaped by the program or instructed by the experimenter. 

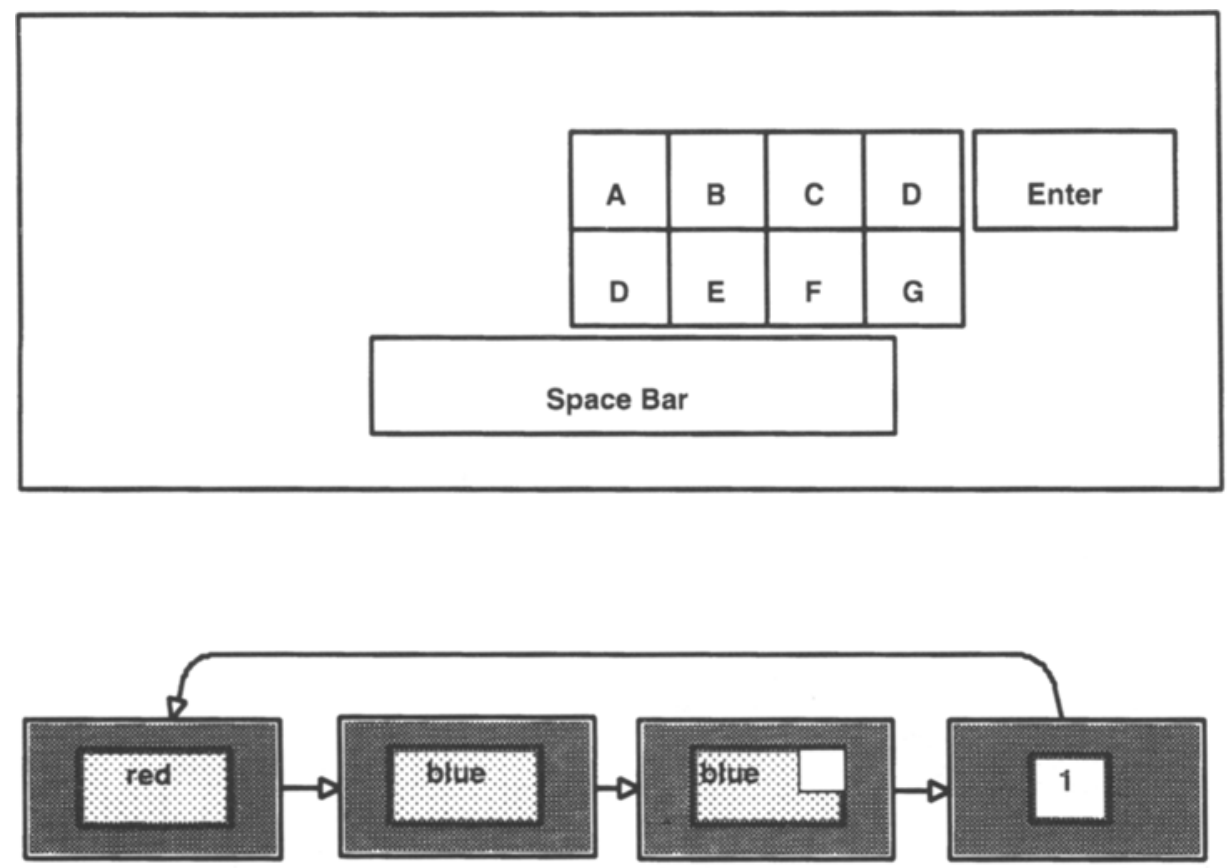

(A)

(B)

(C)

(D)

Figure 1. The top panel shows experimental keyboard with mask covering all keys except the space bar, the Enter key, and eight character keys, labeled here for exposition purposes as ABCDEFGH. In the experiment, the keys had their original labels: $k 1$; ' $\mathrm{m}$, . / The bottom panel shows successive screen displays during shaping trails that required pressing the space bar, character keys, and the Enter key.

One hundred points were earned in each of two subsequent maintenance phases.

Preliminary instructions. The subjects signed a general consent form, which outlined the purpose of the experiment, and were read the following instructions:

Your task is to earn points by generating keypress sequences. Points for correct sequences will appear on the screen. Please only press the eight white (character) keys, the Enter key and the space bar. Also, please only use one hand to press. During the experiment, the kind of pressing that will earn points may change. If this occurs, you will have to alter your pressing patterns. The experiment consists of three trial blocks separated by two short breaks. When the words 'Please leave the room' appear on the screen, call me to set up the computer for the next trial block.

Acquisition. Table 1 presents the step-by-step requirements during shaping for all groups. Each step gives the minimum keypress sequence required for reinforcement. Ten points were earned at each step during acquisition. In the Three-Shape and Three-Instruct groups, referred to in the table as Three-Step, 10 points were earned for pressing at least: (1) the Enter key [E]; (2) the space bar [S] and the Enter key; (3) the space bar, 10 character keys, and the Enter key. In the Six-Shape and Six-Instruct groups, referred to as SixStep, the first three steps were pressing at least the: (1) the Enter key; (2) one character key, the Enter key; (3) the space bar, one character key, the Enter key. The minimum number of character keys then increased from 1 to 3 to 6 and, finally, to 10 .

After this, all the groups earned an additional 40 points for the target sequence. Total overall points in the Three-Step groups was 70 , and in the Six-Step groups, 100. However, total points earned by all groups for the target was the same, 50 . All trials, successful or not, were included in the analyses.
During acquisition, when there was a change in response requirement, the shaped groups had to generate the correct adjustment to the change. The instructed groups were told exactly how to adjust to the requirement change. The experimenter sat with each subject and specified the minimum requirement at each step. For example, at the last step, the instructions were: "Now you have to press the space bar, at least 10 white (character) keys and the Enter key." When 10 points were earned for the final step, the experimenter said, "Now you're on your own," and left the room.

Maintenance. In the two remaining phases of the experiment, 100 points were earned for the target response specified in the last acquisition step.

\section{Measurements}

Three aspects of behavior were analyzed. These were the number, location, and timing of responding. Number, the aspect of responding on which reinforcement was contingent, was calculated by counting how many character keys were pressed on a trial. Location was defined as which character keys were pressed (e.g., $\mathrm{ABCD}$ ). Timing was defined as time between keypresses.

Timing definition. Timing follows Weber's law, which means that, if person A generates longer intervals than person $\mathrm{B}$, a discriminable, or just noticeable, difference (JND) between intervals will be larger for $A$ than for $B$. Consequently, two intervals perceived as different by B might seem the same to A. Weber's law for time implies that the variability in timing should increase with the mean of the interval being timed (Gibbon, 1977). In our data, across all subjects, the correlation between the mean and standard deviation of the time between keypresses was $.48(p<.01)$, making it appropriate to analyze time for each individual relative to their own scale. This was done for individuals in the following manner. Interkeypress times 
Table 1

Step-by-Step Requirements During Shaping

\begin{tabular}{llllllllll}
\hline & & \multicolumn{1}{c}{ Steps } \\
\cline { 2 - 9 } Groups & 1 & 2 & 3 & 4 & 5 & 6 & 7 & 8 & 9 \\
\hline Experiment 1 & & & & & & & & \\
Three-Step & E & SE & S10E & & & & & & \\
Six-Step & E & $1 E$ & S1E & S3E & S6E & S10E & & & \\
Experiment 2 & & & & & & & & & \\
Three-Step & E & SE & S10E & & & & & & \\
Nine-Step & E & $1 E$ & S1E & S2E & S4E & S5E & S7E & S8E & S10E \\
Experiment 3 & & & & & & & & & \\
BigEarly & E & $1 E$ & S1E & S8E & S9E & S10E & & & \\
BigLate & E & $1 E$ & S1E & S2E & S3E & S10E & & & \\
Moderate & E & $1 E$ & S1E & S4E & S7E & S10E & & & \\
\hline
\end{tabular}

from the final training step (when the target sequence was required) to the end of training were examined to identify the time below which $90 \%$ of each subject's interkeypress times fell. Bin size was calculated by dividing the selected interval by 4 . For example, if the time below which $90 \%$ of interkeypress times was $520 \mathrm{msec}$, each bin was $130 \mathrm{msec}$. In this case, all intervals between 0 and $130 \mathrm{msec}$ would be sorted into Interval 1 , those between 131 and $260 \mathrm{msec}$ into Interval 2, and so forth. In all cases, more than four bins were required because, although bin size was based on intervals below $90 \%$, intervals up to the 95 th percentile were sorted into the selected bin size. All intervals higher than the 95 th percentile were placed in a single bin. Number of bins ranged from six to nine.

Variability measures. Uncertainty metrics, derived from information statistics (Attneave, 1959; Miller \& Frick, 1949), were used to measure variability. Uncertainty is a function of the likelihood of possible outcomes. Since outcomes may be conceived of as separate events and sequences of events, variability can stem from changes in the events themselves or in their organization. Separate events are referred to as components. For example, one potentially important aspect of responding in the current procedure was the distribution of specific keys that are pressed (ABCDEFGH). Component uncertainty reflects the likelihood of each individual key location. If all components are equally likely, uncertainty is maximal. Departures from equal probabilities among possible outcomes reduce uncertainty. Sequential uncertainty reflects the likelihood of each key following each other key. The question is: if the A key is pressed, how likely is the next press to be on the A, B, C, or D key? The more orderly the sequence of components is, the lower the uncertainty will be.

Figure 2 is a simplified example showing why it is necessary to separately examine component and sequential uncertainties. The top and middle panels show forward transition probabilities between the same four keys (ABCD). The probabilities represent the likelihood of each location, given the location that preceded it.
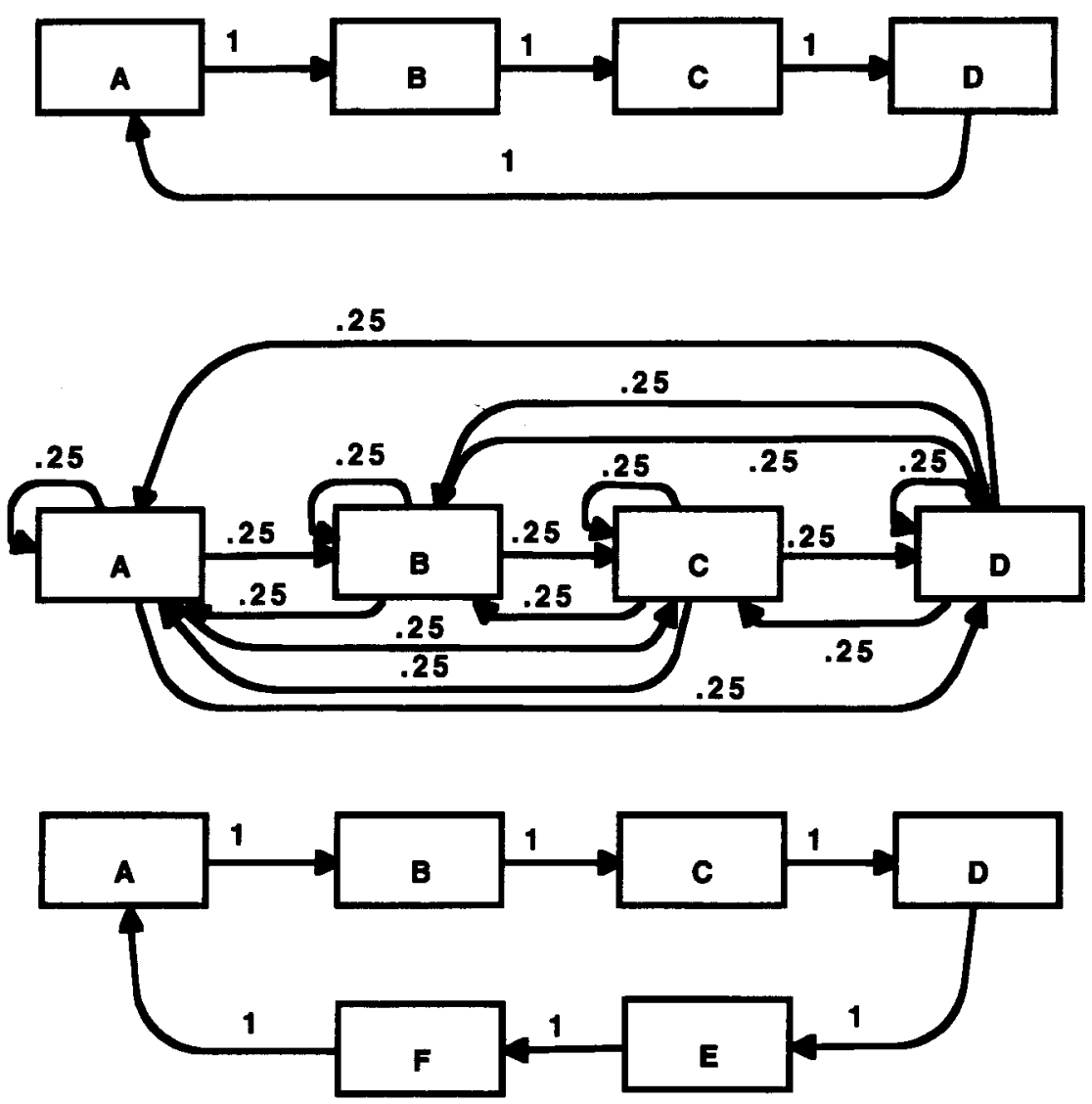

Figure 2. Hypothetical forward transition probabilities during 10 keypressing trials during which each key is pressed once per trial. In the top and bottom panels, the sequence of keypresses is invariant over trials. In the middle panel, the sequences vary so that, over trials, each key is followed at least once by each other key. 

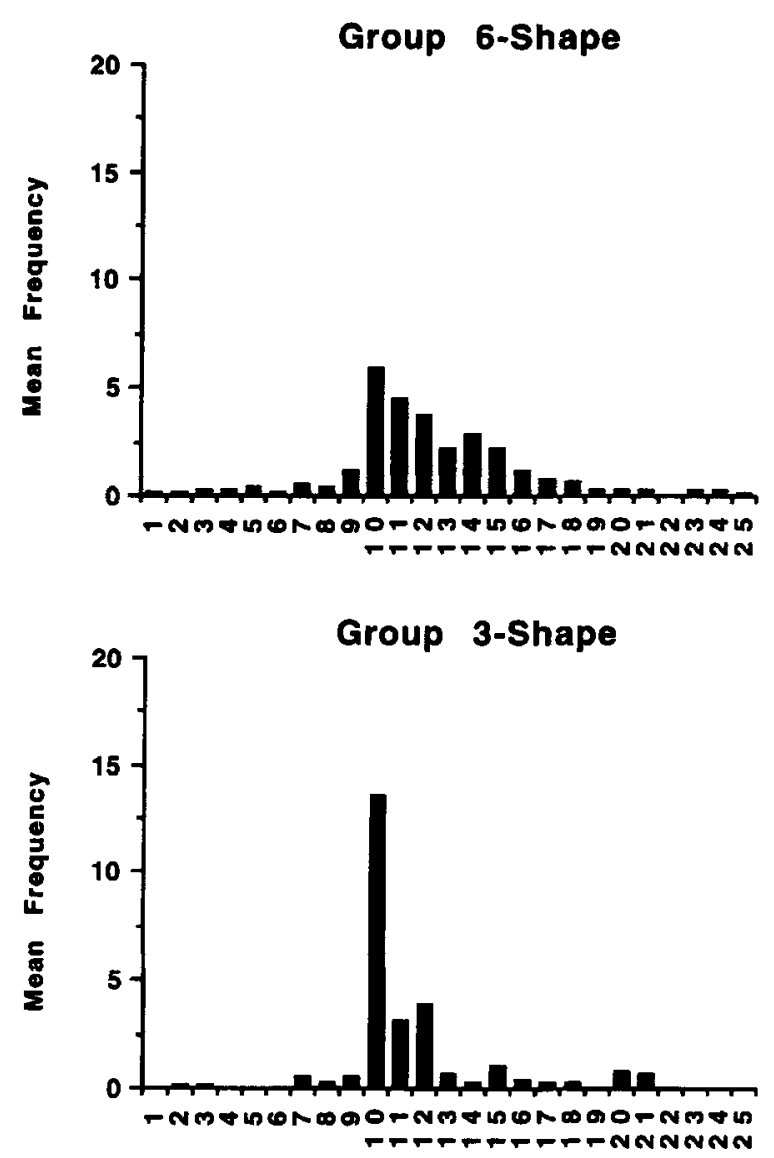
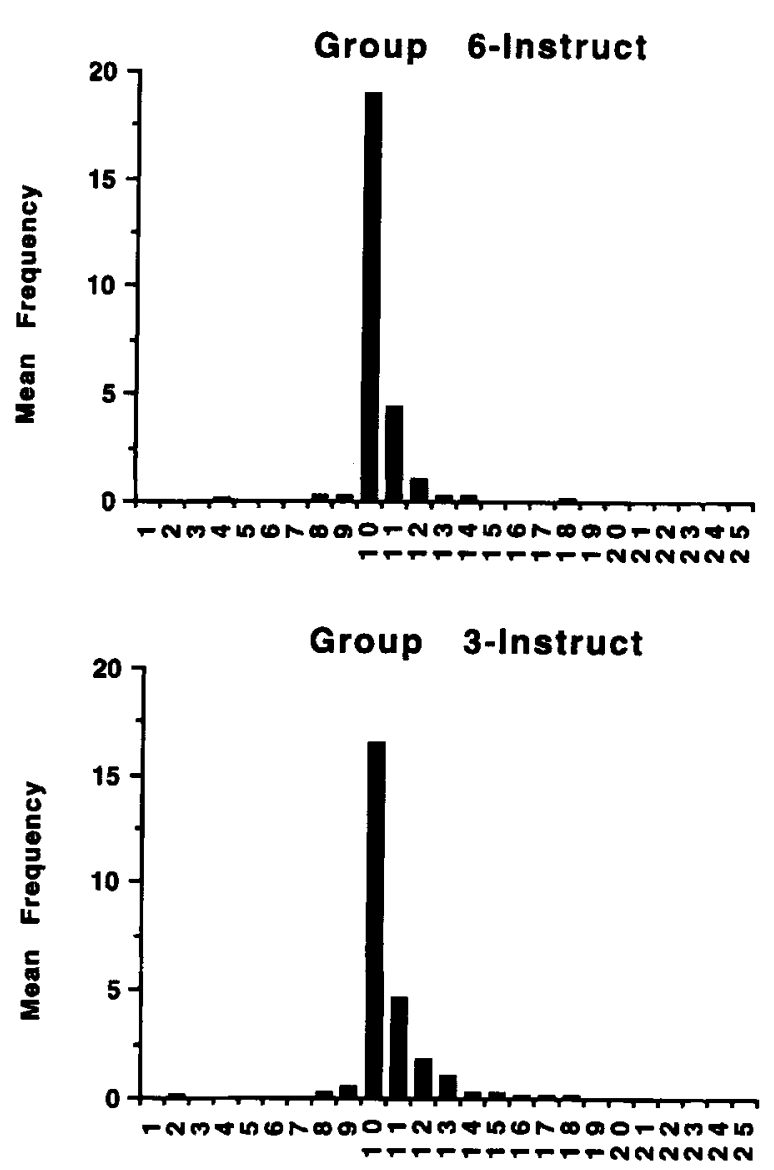

Figure 3. Mean fequency distributions for mean number of responses for all groups at the end of training: Experiment 1.

Since the probability of each location is the same (.25), component uncertainties are identical (2). However, sequential uncertainties are different. In the top panel, each location goes to one other location. Since we are never uncertain what the next key location will be, sequential uncertainty is 0 . In the middle panel, every location goes to every other location. Since there is less certainty about the next location, sequential uncertainty is higher (2.0).

The bottom panel depicts a condition in which the subject presses six of the eight keys. Now there is more uncertainty about which key will be pressed, and this is reflected in increased component uncertainty (2.6). In this example, as in the top panel, each key leads to only one other key, and the sequential uncertainty is 0 .

Comparing the top and middle panels shows that equal component uncertainty does not imply equal sequential uncertainty. Comparing the top and bottom panels shows that equal sequential uncertainty does not imply equal component uncertainty. Thus, both measures are necessary for a variability analysis.

To calculate component uncertainty, the formula

$$
U(\mathrm{R})=-\sum_{n=1}^{N} P(\mathrm{Rn}) \log _{2} P(\mathrm{Rn})
$$

was used, where $U(\mathbf{R})$ is the uncertainty in the set of current responses; $P(\mathrm{Rn})$, the probability that a given response occurred; $N$, the total number of components; and $n$, the individual components that go into $N$. Sequential uncertainty was calculated, using the formula

$$
U[\mathrm{R} /(\mathrm{R}-1)]=U(\mathrm{R}, \mathrm{R}-1)-U(\mathrm{R}-1),
$$

where $U(\mathrm{R} / \mathrm{R}-1)$ is the uncertainty in the joint distribution of current and immediately prior responses.
Statistical analyses. To see how groups differed in variability, one-way analyses of variance (ANOVAs) were run on data from the end of training (Trials 226-250). Fisher's LSD test was used for post hoc comparisons. A significance level of $p<.05$ is used throughout the paper. All responses, whether reinforced or nonreinforced, were included. To determine whether variability was maintained or declined during maintenance, three blocks of 25 reinforced trials taken from early, middle, and late in training were analyzed. These blocks represent responding immediately after acquisition (26th to 50 th reinforcer), in the middle (126th to 150 th reinforcer), and at the end (226th to 250 th reinforcer) of training. The data were subjected to ANOVAs with repeated measures on the blocks factor.

\section{Results}

\section{At the End of Training}

One question of interest was whether different acquisition procedures produce different final levels of variability. To answer this question, responding was examined during the last 25 reinforced trials.

Number. Figure 3 presents mean frequency distributions for number of character keypresses in a sequence for all groups during the last 25 trials. These distributions include both correct and incorrect sequences. Notice that most distributions skew to the right, reflecting the contingency that only rewarded 10 or more presses.

In the Three-Shape, Three-Instruct, and Six-Instruct groups, number of presses clustered around the target. Per- 


\section{Component Uncertainty}

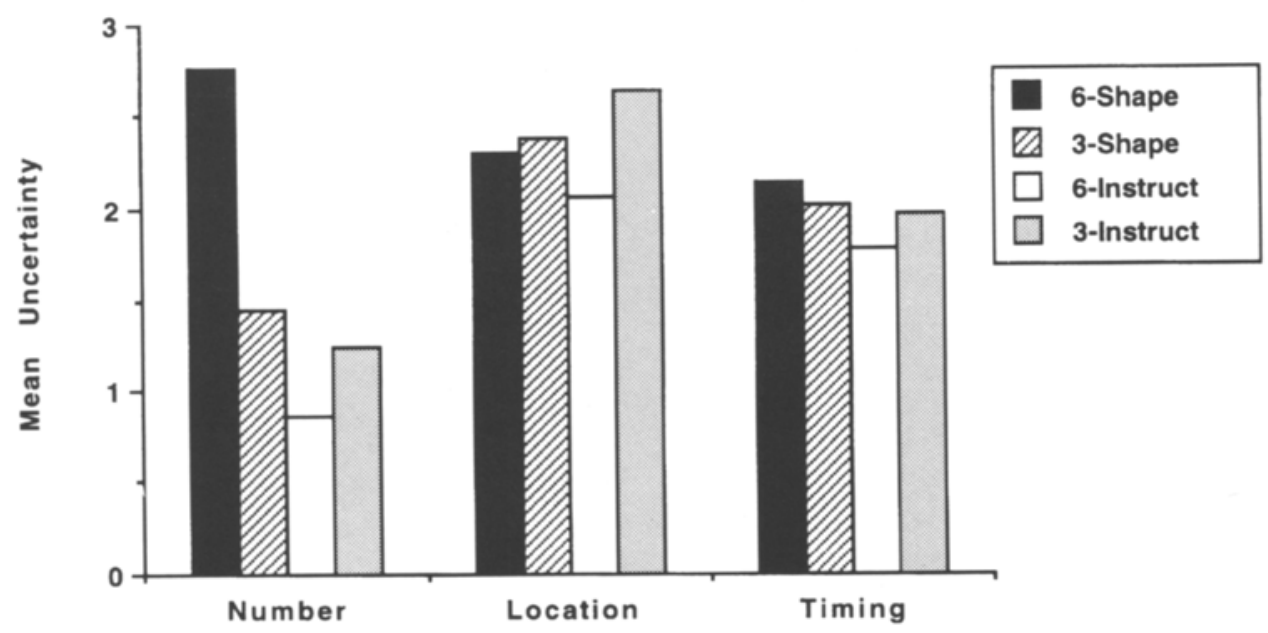

\section{Sequential Uncertainty}

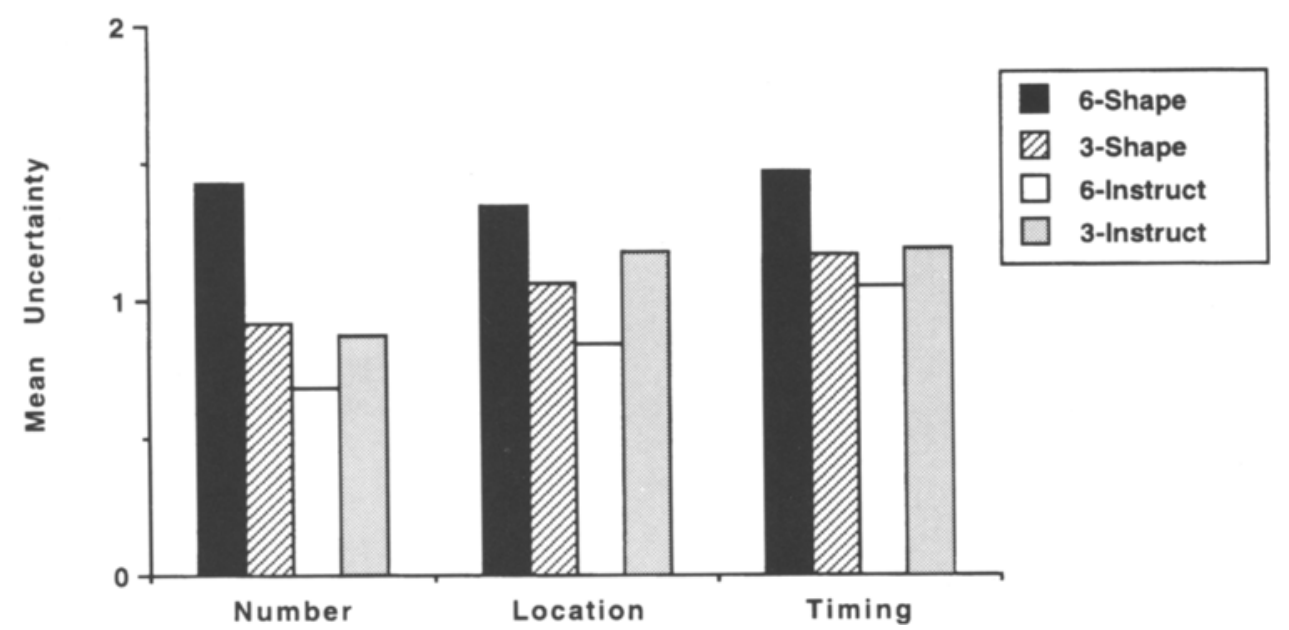

Figure 4. The top panel shows mean component uncertainty, and the bottom, mean sequential uncertainty in number, location, and timing for all groups at the end of training: Experiment 1.

centages at 10 were: Three-Shape, $52 \%$; Three-Instruct, $65 \%$; Six-Instruct, $75 \%$. Percentages over 10 were: ThreeShape, 43\%; Three-Instruct, 32\%; Six-Instruct, 23\%. In contrast, the Six-Shape group showed greater spread and skew in its distribution. Only $21 \%$ of numbers of responses in the Six-Shape group were at 10;68\% were over 10 .

The leftmost sets of bars in Figure 4 present mean component (top panel) and sequential (bottom panel) uncertainties for number at the end of training. There were significant group differences in both measures [component uncertainty: $F(3,36)=11.703$; sequential uncertainty: $F(3,36)=7.597$ ]. Fisher's LSD tests showed that the group shaped in six steps (Six-Shape) was significantly more variable than the others in number of responses.
Location and timing. The middle and right sets of bars in Figure 4 show component and sequential uncertainty for the two nonreinforced aspects of behavior. There were no between-group differences.

\section{Changes During Maintenance}

A second question was whether variability changed after acquisition. Three-way ANOVAs with method (shape vs. instruct), step (three vs. six), and repeated measures on block $(1,2,3)$ as factors showed main effects of block in both location and timing. Component uncertainty declined in location $[F(2,35)=5.699]$. Component $[F(2,35)=3.936]$ and sequential uncertainties $[F(2,35)=$ $3.639]$ declined in timing. There were no changes in uncertainty for number. 


\section{Summary}

At the end of training, there was no main effect of type of training (shaping or instruction) or of number of training steps (three or six) on variability. Rather, the group shaped in six steps (Six-Shape) was more variable than the other three groups in number.

In all groups, once the target was acquired, variability in number, the aspect of behavior on which reward was contingent, did not change. In contrast, variability declined in both location and timing.

\section{Discussion}

The present results partially replicate work (e.g., Galizio, 1979) indicating that, even with the same target, shaped behavior is more variable than is instructed. Both instructed groups had more completely specified instructions, which told them exactly how to vary. Both shaped groups had to figure this out. Nonetheless, the group shaped in three steps was low in variability, like the two instructed groups and unlike the group shaped in six steps.

The results also partially support the idea that variability depends on number of training steps. More steps generated more variability in the shaped groups.

The remaining experiments were designed to systematically examine variables that might account for the differences between the low- and high-variability shaped groups. Since the Six-Shape group had both more shaping steps and smaller step sizes (differences in minimum required presses between steps), Experiments 2 and 3 assessed how number and size of shaping steps affect variability.

\section{EXPERIMENT 2 \\ Effect of Increasing the Number of Shaping Steps}

Experiment 1 showed that subjects shaped in six steps were more variable than those shaped in three, raising the possibility that, when a shaping procedure is used, more steps do generate greater variability. In Experiment 2, this idea was investigated by shaping the same target (space bar, at least 10 character keypresses, Enter key) in three and nine steps. These groups are referred to as ThreeStep and Nine-Step. If the number of steps is important, the Nine-Step group should be more variable than the Three-Step group.

\section{Method}

Subjects

The subjects were 20 undergraduates ( 17 female, 3 male).

\footnotetext{
Apparatus and Stimuli

The apparatus and stimuli were the same as those described in Experiment 1.

\section{Procedure}

The procedure is described in Experiment 1. Table 1 shows the step-by-step minimum keypress requirements during shaping for the two groups. Ten points were earned at each step. In the ThreeStep group, 10 points were earned for pressing at least: (1) the Enter
}

key [E]; (2) the space bar [S] and the Enter key; (3) the space bar, 10 character keys, and the Enter key. In the Nine-Step group, 10 points were earned for pressing at least: (1) the Enter key; (2) one character key and the Enter key; (3) the space bar, one character key, and the Enter key. The minimum number of required character keys then increased to 2 , to 4 , to 5 , to 7 , to 8 , and finally to 10 .

\section{Results}

\section{At the End of Training}

Number. Figure 5 presents mean frequency distributions for number of character keypresses in a sequence during the last 25 reinforced trials. In both groups, responding was greatest at the target and skewed to the right, above the target. Percentages at the target (10) were: ThreeStep, $31 \%$; Nine-Step, $30 \%$. The groups did not differ in component or sequential uncertainty for number.

Location and timing. There were no between-group differences in component or sequential uncertainty for location or timing.

\section{Changes During Maintenance}

There was no decline in variability in number of character keypresses. However, one-way ANOVAs with group as a factor and repeated measures on block showed that sequential uncertainty declined in both location $[F(2,17)=$ $3.937]$ and timing $[F(2,17)=4.279]$.

\section{Summary}

There were no differences between the Three-Step and the Nine-Step groups at the end of training. However, as can be seen in Experiment 1, once the target was acquired, variability did not decline in number, the aspect of responding on which reinforcement was contingent, but did decline in location and timing.

\section{Discussion}

Contrary to the prediction that more shaping steps would generate greater variability, the Nine-Step group was not more variable than the Three-Step group. In addition to suggesting that number of steps, per se, is not a critical variable in determining variability, this also indicates that the order in which the elements of the target were acquired was not responsible for the differences between the Three- and Six-Shape groups from the previous experiment.

At Step 2 (see Table 1), only the space bar and the Enter key were required in the group with three shaping steps, whereas the space bar, at least one character key, and the Enter key were required in the group shaped with six steps. Despite maintaining this different order, the Three- and Nine-Step groups in Experiment 2 did not differ from each other. Thus, neither the larger number of steps nor the particular order was sufficient for generating greater variability in the Six-Shape, as compared with the Three-Shape, group in Experiment 1.

Although it appears that number of steps, per se, is not a big influence on variability, the groups differed not only in number but also in size of shaping steps. Recall that 

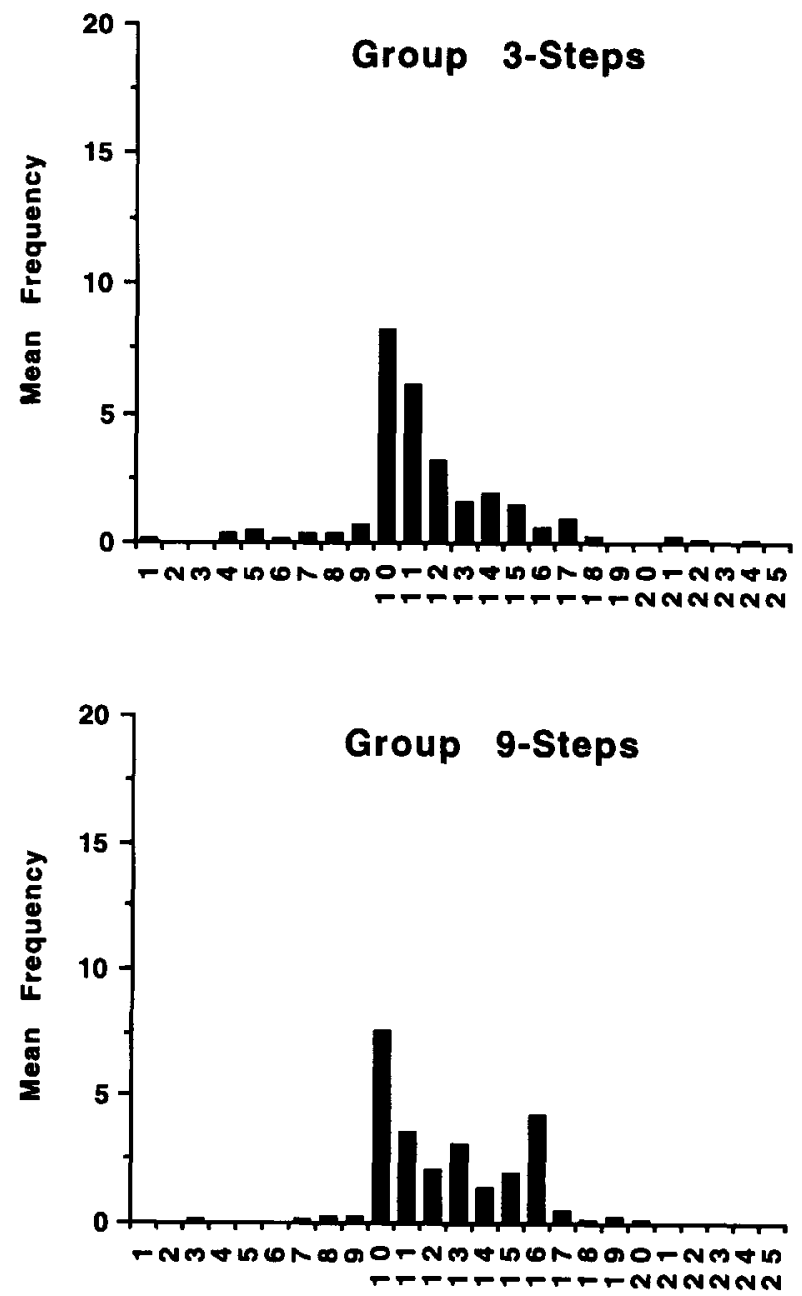

Figure 5. Number distributions for the three- and nine-step groups at the end of training: Experiment 2.

step size refers to successive increases in minimum required keypresses. Given the same target, as the number of steps increases, average step size decreases. In the Three-Step group, the minimum requirement went from 0 to 10 , making the step size +10 . In the Nine-Step group, all steps were small, alternating between +1 and +2 . Since Experiment 2 confounded the manipulation of step size and number, conclusions need to be tempered by acknowledging the possibility that the interaction of these variables may have masked their effects. The goal of Experiment 3 was to examine the effect of changing step size with number of steps held constant.

\section{EXPERIMENT 3 \\ Effect of Order and Size of Steps With Number of Steps Constant}

Just as the Three- and Nine-Step groups in Experiment 2 differed in step size, so did the Three- and SixShape groups in Experiment 1. The Six-Shape group was exposed to increments ranging from one to four additional responses at each step during shaping. The ThreeShape group was exposed to a single increment of 10 . Thus, it is possible that exposure to a large step produces low variability. In Experiment 3, this possibility was investigated by comparing subjects trained with a maximum step size of +7 with subjects trained with a maximum step size of +3 . These maxima were implemented in a six-step procedure with a target of 10 keypresses. Within these constraints, the largest possible step size is +7 ; this requires that the remaining three steps be +1 each. The smallest maximum step size is +3 ; this condition can only be implemented with three shaping steps of +3 and one step of +1 .

To see whether variability is affected by when in shaping a particular size steps occurs, the large step $(+7)$ procedure was implemented in two ways. Since the ThreeShape group of Experiment 1 had its large step at the start of shaping, low variability may be specific to a large early step. In the present experiment, the large step was introduced early or late in shaping. In the BigEarly group, character press requirements went from 0 to 1 to 8 to 9 to $10(+1,+7,+1,+1)$. In the BigLate group, they went from 0 to 1 to 2 to 3 to $10(+1,+1,+1,+7)$. In the third group (Moderate) with the maximum step size of +3 , requirements went from 1 to 4 to 7 to $10(+1,+3,+3,+3)$.

If a large step size is sufficient to decrease variability, the BigEarly and BigLate groups should be less variable than the Moderate group. If a large step early in shaping decreases variability, then the BigEarly group should be less variable than the BigLate or Moderate groups.

\section{Method}

Subjects

The subjects were 30 undergraduates ( 22 female and 8 male).

\section{Apparatus and Stimuli}

The apparatus and stimuli were the same as those in Experiment 1 .

\section{Procedure}

The procedure is described in Experiment 1. Table 1 shows each group's step-by-step minimum keypress requirements during shaping. Ten points were earned at each step. The first three steps were the same in all groups. Ten points were earned for pressing at least: (1) the Enter [E] key; (2) one character key and the Enter key; (3) the space bar [S], one character key, and the Enter key. In the BigEarly group, the minimum character key requirement then increased to 8 , to 9 , and finally to 10 . In the BigLate group, the requirement increased to 2, to 3, and then to 10. In the Moderate group, it increased to 4 , to 7 , and then to 10 .

\section{Results}

\section{At the End of Training}

Number. Figure 6 shows mean frequency distributions for number of character keypresses in a sequence for all groups during the last 25 trials. As in Experiments 1 and 2, distributions skewed right, above the target, in all groups. The highest percentage occurred at the target (10) for the BigLate (38\%) and Moderate (31\%) groups. 

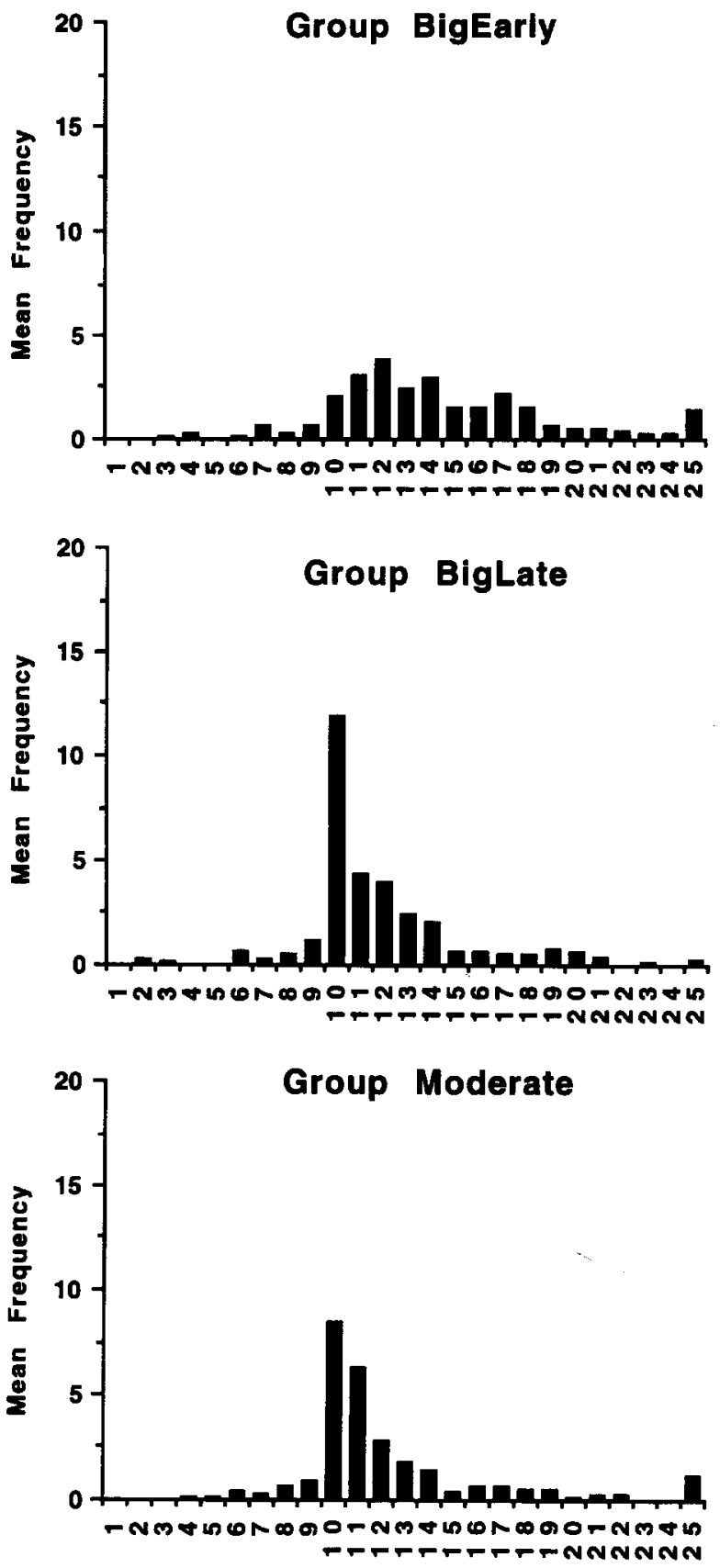

Figure 6. Number distributions for the BigEarly, BigLate, and Moderate groups at the end of training: Experiment 3.

The flattest distribution was seen in the BigEarly group, for whom only $7.5 \%$ of trials generated exactly the targeted number of presses (10).

The leftmost sets of bars in Figure 7 show mean component (top panel) and sequential (bottom panel) uncertainty for number of responses at the end of training. Since the one-way ANOVA with group as a factor approached significance $[F(2,27)=2.97, p=.06]$, Fisher's LSD test was used to compare the groups separately. The BigEarly group was more variable than either the BigLate or the Moderate group, which did not differ from each other in variability. Though the BigEarly group did not differ from the others in sequential uncertainty, it did exhibit many more different transitions in number of responses from one trial to the next. The mean number of unique transitions (occurring a single time) for the BigEarly, BigLate, and Moderate groups were 21.4, 16.1, and 11.9, respectively. Because so many transitions for the BigEarly group occurred a single time, a data structure with many high probability (1.0) transitions occurred, and low sequential uncertainty was generated. (See Figure 2, bottom panel, for an example of this kind of result.)

Location and timing. The middle and right sets of bars in Figure 7 show component and sequential uncertainty for location and timing. The only between-group difference occurred in location $[F(2,27)=3.32]$. Fisher's LSD test showed that the BigEarly group was more variable in sequential uncertainty than the other two groups, which did not differ from each other.

\section{Changes During Maintenance}

Variability did not decline in either response number or timing. However, both component $[F(2,26)=5.867]$ and sequential $[F(2,26)=3.648]$ uncertainties declined in location.

\section{Summary}

At the end of training, there was greater component uncertainty for number in the BigEarly group than in the other two groups. The BigEarly group was also most variable in sequential uncertainty for location. There were no between-group differences in variability of timing.

In all groups, component and sequential uncertainty remained unchanged in number and timing but declined in location.

\section{Discussion}

Experiment 3 shows that sustained levels of variability depend on early histories (see, e.g., Stokes, 1995a). However, contrary to prediction, a large early step generated higher variability than did an equally large later step or moderate size steps. Thus, the size of a step, per se, does not determine variability. What seems to be important is the particular sequence of steps. The difference between the BigEarly and the BigLate groups suggests that a large early step may increase variability. However, the low variability of the Three-Shape group in Experiment 1 resulted from a single large step. Thus, a large step alone is not sufficient for generating high variability. Perhaps a large step following a reinforced small step (as in the BigEarly group) is critical for higher variability. Additionally, this step must occur relatively early in acquisition.

There is evidence from other tasks that supports the idea of a critical period early in training for establishing variability levels. Rats shaped with a more restrictive topographic constraint were more variable than those shaped with a less restrictive constraint, even when the 


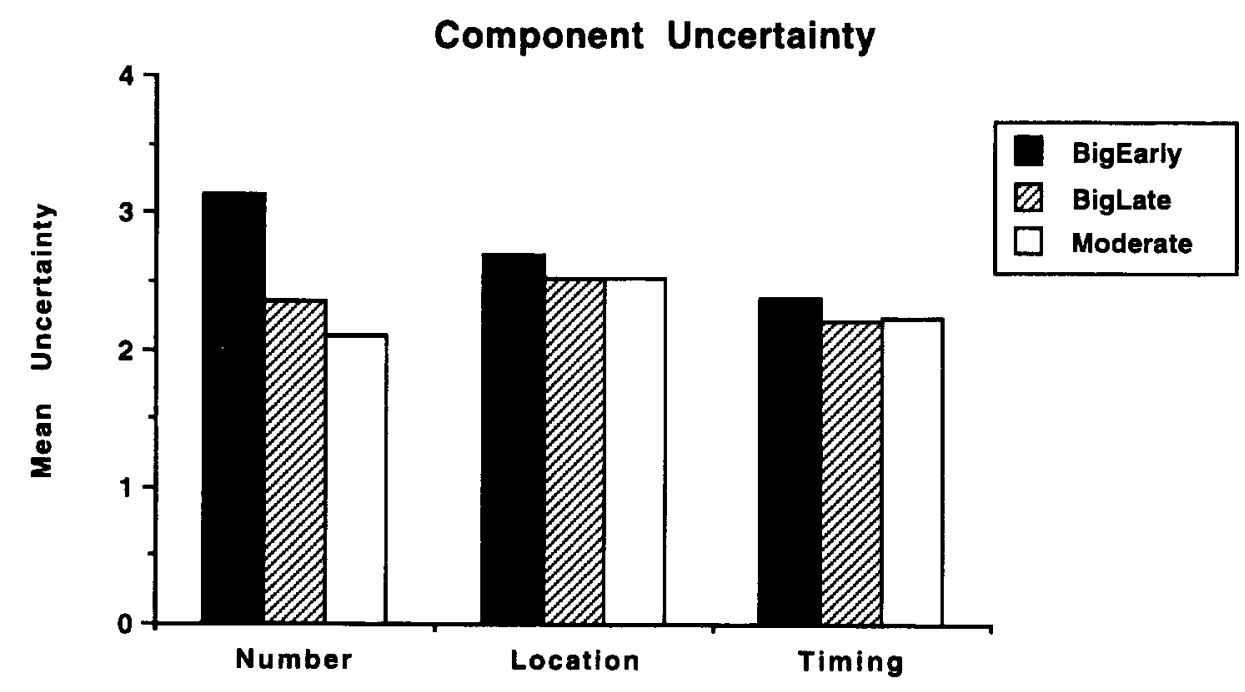

\section{Sequential Uncertainty}

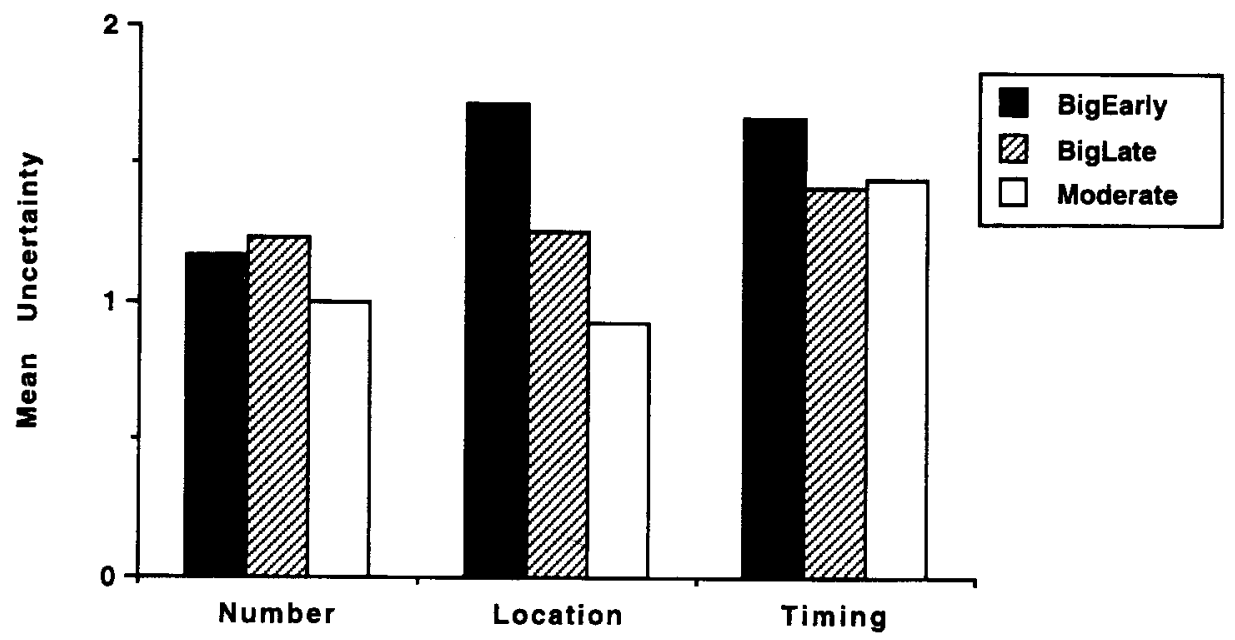

Figure 7. The top panel shows mean component uncertainty and the bottom, mean sequential uncertainty, in number, location, and timing for all groups at the end of training: Experiment 3.

contingencies were switched later in training (Stokes, 1995a). Similarly, college students exposed to an initially high lag requirement were more variable throughout training than students subjected to the high lag requirement later in training (Stokes, in press).

\section{GENERAL DISCUSSION}

\section{Findings}

In the present studies, the effects of different acquisition procedures on sustained levels of response variation in several aspects of responding were examined. Across all procedures, variability was maintained in the reinforced aspect of responding (number) but declined in one or both aspects not targeted for reinforcement (location and timing). This finding supports the idea that, like persistence (Eisenberger, 1992), a habitual level of variabil- ity will be acquired and maintained in the response dimensions targeted by the explicit reward contingency (Stokes, 1995a).

The three experiments systematically eliminated variables potentially responsible for acquisition of such variability levels. The initial hypothesis, that variability was a simple function of number of training steps, was not supported. Rather, the sequence of training steps proved important. In Experiment 3, it was shown that a large early step led to high variability, whereas an identical step introduced later in shaping did not. It should be noted, however, that a large early shaping step does not invariably lead to high variability. In Experiments 1 and 2 , introducing the final requirement in one training step $(+10)$ generated relatively low variability. In Experiment 3 , when a large early step $(+7)$ followed a reinforced smaller step $(+1)$, high variability was induced. 


\section{Hypotheses}

This pattern of results suggests that, like other aspects of behavior, including persistence (Eisenberger, 1992), rate (Weiner, 1964), and form (Stokes \& Balsam, 1991), variability depends on early reinforcement history.

There are two ways in which reinforcement can affect variability. First, different reinforcement rates generate different degrees of response variation. Variability is higher with intermittent than with continuous reinforcement (Eckerman \& Lanson, 1969). Thus, low levels of reward generate greater variability than high ones, and shifts in reinforcement generate shifts in variability. Second, variability is itself a reinforceable aspect of behavior (see, e.g., Page $\&$ Neuringer, 1985). These two processes may account for the enduring effects of early reinforcement history on variability. It is possible that, in different acquisition procedures, the switch from one step to another alters variability by changing reinforcement rates and that these different levels of variability are differentially reinforced.

Alternatively, what gets acquired early in training may not be sustained levels of variability, per se. Rather, differences in the specific repertoires that are acquired with different procedures may be responsible for observed variability levels. For example, acquisition procedures might differ in the number of correct response variants that are directly strengthened, giving rise to maintained differences in variability. In a similar vein, different acquisition procedures might induce qualitatively different response strategies. For example, correct responses in the current procedure could be generated by counting the number of responses or by responding at a fixed rate for an appropriate duration. Such qualitatively different strategies would be likely to sustain different variability levels. All of these hypotheses are considered in more detail below.

\section{Variability as a Function of Reinforcement Rate and Differential Reinforcement}

To evaluate the first hypothesis, that variability levels were selected, two things were analyzed: intermittency of reinforcement, calculated as percentage of all reinforced trials (Total) and reinforcement of variability, measured by reinforced transitions to different numbers of responses from trial to trial (Diff). For example, such a transition would occur if, on trial $n-1, X$ character keys were pressed, and on the subsequent trial $n$, any number of keys other than $X$ were made (but greater than 10 ). Reinforcement history was examined during the time indicated by Experiment 3 as critical for establishing different variability levels. This seems to be when all distinct elements of the target sequence (e.g., space bar, one character key, Enter key) are first required. The following analyses thus includes the period in which the first 40 reinforcers were earned for sequences that included the space bar, at least one character key, and the Enter key. For all groups, this period starts at the third step of Table 1 .

The data were analyzed separately in blocks of 10 reinforced trials. The blocks included nonreinforced trials as well. Stepwise regressions with Total, Diff, and their interaction (Total ${ }^{*}$ Diff) as predictors of component uncertainty for number of responses at the end of training were run for each block. Significant results were only obtained for the block that included the 31 st to the 40 th reinforced trials. For the four Six-Step and the one NineStep groups, this was Step 4, when the minimum number of required keypresses increased from 1. For the ThreeStep groups, it included the 10 reinforcers after Step 3, when the final target of 10 presses was mandatory.

The stepwise regression for all groups showed that only Diff contributed to the significant result $[F(2,87)=$ $8.737]$. Figure 8 shows the relation between mean terminal component uncertainty for number and the percentage of reinforcers obtained for a number that differed from the preceding one. The groups are placed on the $a b-$ scissa in order of increasing uncertainty. Note that the highest percentage of reinforced transitions to different sequence lengths occurs in the most variable group (BigEarly) and the lowest percentage is seen in the least variable (Six-Instruct). There are two obvious exceptions to this pattern. The Three-Shape and Three-Step groups have high percentages Diff during the fourth step but low terminal variability. These groups differed from the others in their overall rate of reinforcement during the early steps of acquisition. They had only around $20 \%$ of their trials reinforced in Step 3 and about $60 \%$ reinforced in Step 4, compared with an average of $83 \%$ in both steps for the other groups. This suggests that early high variability in the Three-Shape and Three-Step groups was due to highly intermittent reinforcement rates (Eckerman \& Lanson, 1969). Regardless, however, of the reason why these groups were variable, the low percentage of reinforced sequences during the fourth step means that there was less strong selection for variability than in the other groups, whose early reinforcement for variability was predictive of their terminal variability levels

The stepwise regression was rerun on the data excluding the Three-Step and Three-Shape groups. The overall regression was again significant $[F(2,67)=13.163]$. This time, both variables were significantly related to terminal variability [Total, $t(67)=-2.21$; Diff, $t(67)=2.94$ ]. This analysis is consistent with the hypothesis that terminal levels of variability are related to the strength with which variability is selected early in training. The groups that were most selectively reinforced for variable behavior during Step 4 are the most variable at the end of training. This analysis supports the idea that implicit variability criteria are embedded in training procedures that do not directly reinforce variability (Neuringer, 1993; Stokes, 1995b). In this view, individuals learn how variably to do a task, even though reward does not depend on being variable.

\section{Variability as a Function \\ of Repertoire Acquisition}

Competing response habits. As in the differential reinforcement of variability hypothesis, if maintained variability depends on the number of specific variants that 


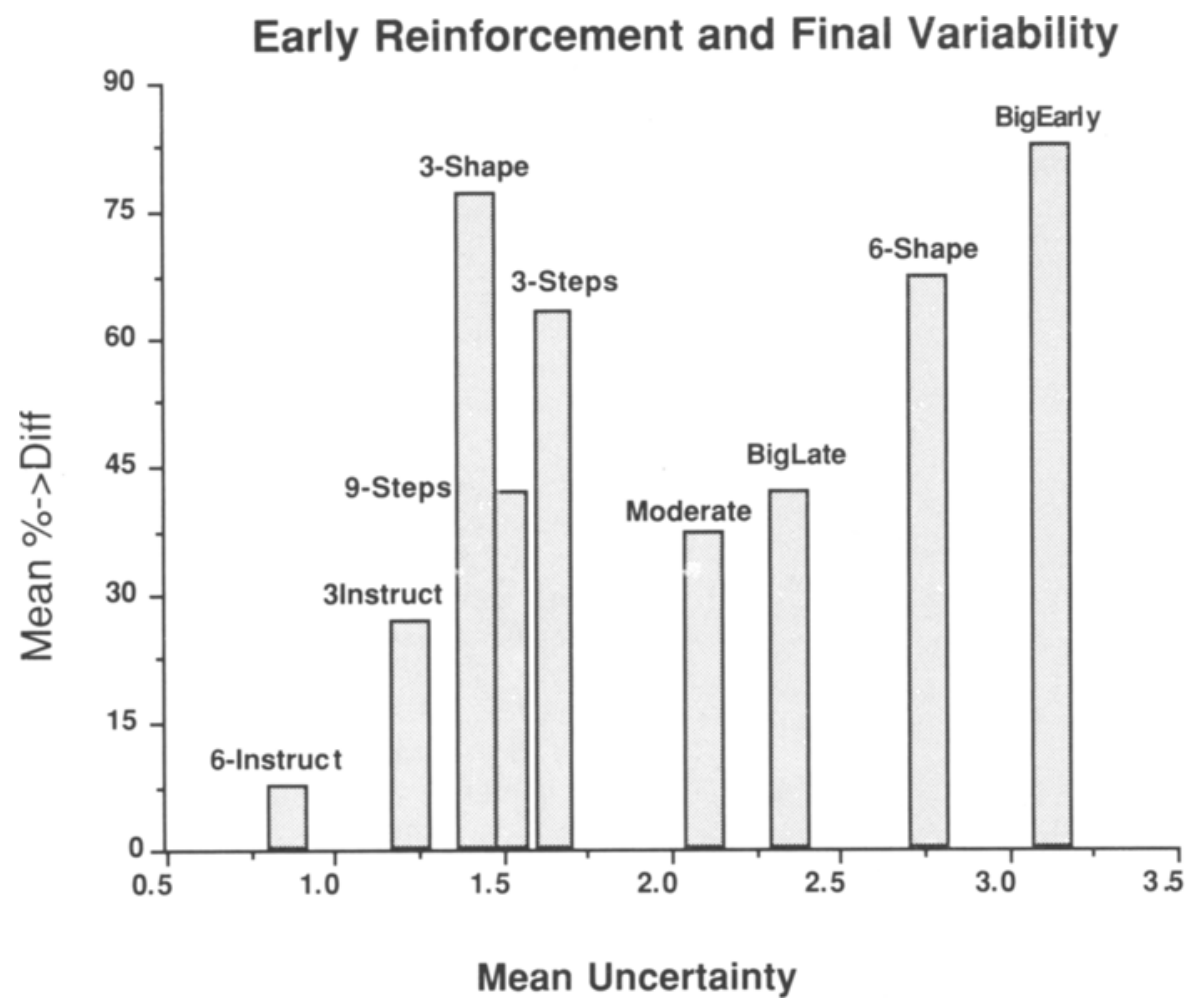

\begin{abstract}
Figure 8. Median percentage of reinforced transitions to different numbers of responses during the 31 st to 40 th reinforced trials. Groups are placed on the $x$-axis in order of increasing terminal variability in number.
\end{abstract}

were reinforced during acquisition, we should find that the most variable groups were reinforced for more variants than the less variable groups. However, unlike the differential reinforcement of variability hypothesis, this hypothesis requires that the specific variants exhibited during maintenance should be those that were strengthened in acquisition. To evaluate the hypothesis that specific responses were differentially reinforced early - and maintained later-in training, we compared the specific variants in the number of presses that each subject emitted at the end of shaping with those they emitted at the end of training. Table 2 shows the mean percentage of variants at the end of training that had not been reinforced during acquisition. The groups in the table are arranged according to variability at the end of training. The most variable (BigEarly) is at the top of the list, and the least variable (Six-Instruct) is at the bottom. It is evident from the analysis that the most variable groups generated many variants that were not reinforced in shaping. Note, too, that the low-variability groups also show a large percentage of variants that were not reinforced during the acquisition phase. Thus, variability is not a simple product of repeating what has been reinforced.

Response size. Another possible mediator of variability differences has to do with the size of an acquired response. In general, there is a positive correlation between the size of a quantity estimate and the variability of the estimate. For example, when rats are required to make a specific number of responses to receive reward, the variance increases with the size of the requirement (Mechner, 1958). Though all of our conditions required a minimum of 10 responses, the subjects in the most variable groups tended to make more responses per reward than did subjects in the less variable groups. The percentage of sequences that included more than 10 responses for the SixShape and BigEarly groups were $68 \%$ and $85 \%$, as compared with $49 \%$ for the remaining groups. In the BigEarly group, 3 subjects had a modal number of responses per sequence larger than 12 . In the BigEarly group, 5 subjects had modes larger than 12. In most of the low-variability groups, the subjects tended to make 12 or fewer responses per sequence. However, one low-variability group (NineStep) had 4 subjects whose modes were greater than 12 responses per sequence. In fact, two of these consistently emitted sequences of 16 responses (larger than anyone in the Six-Shape group, and larger than all but 2 members of the BigEarly group), yet were quite stereotyped. Thus, the variability levels established by the different acquisition procedures reported here are not likely to be mediated by differences in response size.

\section{Variability as a Function of Strategy Acquisition}

Our final hypothesis is that sustained variability levels depend on acquisition of response strategies early in 
Table 2

Mean Percentage of Novel Press Numbers at the End of Training Relative to the End of Acquisition

\begin{tabular}{lc}
\hline \multicolumn{1}{c}{ Group } & Mean \% Novel Press Numbers \\
\hline BigEarly & 70 \\
Six-Shape & 58 \\
BigLate & 46 \\
Moderate & 55 \\
Three-Steps & 54 \\
Nine-Steps & 75 \\
Three-Shape & 46 \\
Three-Instruct & 52 \\
Six-Instruct & 40 \\
\hline
\end{tabular}

training. In this view, strategies that successfully meet early shifts in explicit criteria are differentially reinforced. For example, it is possible for subjects to produce the correct minimum number of responses in a sequence by counting, by emitting presses at a relatively constant rate for a fixed duration, or by following a spatial pattern of keys that meets the reinforcement criterion. These qualitatively different strategies could easily be associated with different levels of variability: a counting or stereotyped location strategy should produce lower variability in number of responses than a strategy involving time estimation.

Additionally, much of the variability observed in problem solving is due to shifts from one to another effective strategy (see, e.g., Siegler, 1995), suggesting that variability is also affected by switching, or not switching, between different strategies. Sustained differences in variability may then be related both to how many different strategies are acquired and to a tendency to switch, or not switch, between them.

Finally, variability could be affected by acquisition of a higher order strategy, such as "be variable while doing this task." Postexperimental interviews with our subjects suggested that some of the acquisition conditions induced this general strategy. Six subjects in the highly variable BigEarly group reported that they had to continue changing their location patterns in order to earn points. No one in either Instructed group offered this analysis. In the Six-Instruct group, 9 subjects said that they counted. In the Three-Instruct group, 5 reported counting. Three others said they pressed each of the eight character keys once and then did 2 more presses on any key-a procedure that yielded exactly 10 presses and that could represent either a pure counting strategy or a combined location and count strategy.

\section{CONCLUSIONS}

The present study extends earlier work (Stokes, 1995a) showing that initial training conditions that do not directly target variability have lasting effects on how differently a task continues to get done. A major implication of this study is that there is a critical period for sustained levels of response variability in the response dimension ex- plicitly targeted for reinforcement. These sustained levels do not depend on response size or on competing habits. Rather, they follow from differential reinforcement of variability early in training.

In the present experiments, the groups in which there was particularly strong selection for variability seem to have a common history. The subjects in these groups were initially successful but met with failure when a large step was introduced early in the acquisition sequence. An initial period of high reinforcement followed by failure (extinction) is likely to induce high variability (Balsam, Paterniti, Zechowy, \& Stokes, 1998). If this increased variability level leads to satisfying the new criterion and reinstating a high reinforcement rate, the variability level will be sustained. Low variability levels will be maintained if early criteria shifts are successively accomplished with little variation or if initially high variability is not differentially reinforced (as in the cases of the Three-Step groups).

There are some interesting parallels between our findings on variability and prior work on persistence (Amsel, 1992) or learned industriousness (Eisenberger, 1992). As with variability, a period of reduced reward is essential for producing high industriousness or persistence. People are more persistent in problem solving when they initially experience failure or difficulty followed by success. They learn to be persistent, even though reward is not contingent on persistence, and maintain high or low persistence in the response dimension targeted for reward (Eisenberger, Masterson, \& McDermitt, 1982). These results are interpreted on the basis of reinforcement of effort. The parallels suggest that the same operations may underlie production of both variability and persistence.

For example, it is possible that a high level of persistence in extinction - when variability increases relative to continuous reinforcement (Antonitis, 1959; Eckerman \& Lanson, 1969; Millenson \& Hurwitz, 1961)—depends on previous acquisition of a highly variable repertoire. Schoenfeld (1968) offered an account of the partial reinforcement extinction effect on the basis of the idea that speed of extinction depends on successfully extinguishing all variants of the reinforced response. If an acquisition procedure generates few reinforced variants, extinction will be more rapid than after a procedure that generates many. Thus, persistence may depend on prior reinforced variability.

\section{REFERENCES}

Amsel, A. (1992). Frustration theory: An analysis of dispositional learning and memory. New York: Cambridge University Press.

Anderson, J. R. (1993). Problem solving and learning. American Psychologist, 48, 35-44.

Anderson, J. R., Conrad, F. G., \& Corbett, A. T. (1989). Skill acquisition and the LISP tutor. Cognitive Science, 13, 467-506.

ANTONITIS, J. J. (1959). Response variability in the white rat during conditioning, extinction, and reconditioning. Journal of Experimental Psychology, 42, 273-281. 
At TNEAVE, F. (1959). Applications of information theory to psychology: A summary of basic concepts. New York: Holt, Rinehart \& Winston. BAER, J. (1994). Divergent thinking is not a general trait: A multidomain training experiment. Creativity Research Journal, 7, 35-46.

Balsam, P. D., Paterniti, A., Zechowy, K., \& Stokes, P. D. (1998). Effects of outcome shifts on behavioral variability. Manuscript submitted for publication.

BowERMAN, M. (1982). Starting to talk worse: Clues to language acquisition from children's late speech errors. In S. Strauss (Ed.), $U$ shaped behavior growth (pp. 101-145). New York: Academic Press.

CARPEnter, T. P., \& Moser, J. M. (1982). The development of addition and subtraction problem-solving skills. In T. P. Carpenter, J. M. Moser, \& T. A. Romberg (Eds.), Addition and subtraction: $A$ cognitive perspective (pp. 9-24). Hillsdale, NJ: Erlbaum.

Eckerman, D. A., \& Lanson, R. L. (1969). Variability of response location for pigeons responding under continuous reinforcement, intermittent reinforcement and extinction. Journal of the Experimental Analysis of Behavior, 12, 73-80.

EISENBERGER, R. (1992). Learned industriousness. Psychological Review, 99, 248-267.

Eisenberger, R., Masterson, F. A., \& McDermit t, M. (1982). Effects of task variety on generalized effort. Journal of Educational Psychology, 74, 499-505.

EISENBERGER, R., \& SELBST, M. (1994). Does reward increase or decrease creativity? Journal of Personality \& Social Psychology, 66, $1116-1127$

GaLizio, M. (1979). Contingency-shaped and rule-governed behavior: Instructional control of human loss avoidance. Journal of the Experimental Analysis of Behavior, 31, 53-70.

GibBon, J. (1977). Scalar expectancy theory and Weber's law in animal timing. Psychological Review, 84, 278-325.

GoETz, E. M., \& BAER, D. M. (1973). Social control of form diversity and the emergence of new forms in children's blockbuilding. Journal of Applied Behavior Analysis, 6, 209-217.

Goldin-Meadow, S., Alibani, M. W., \& Church, R. B. (1993). Transitions in concept acquisition: Using the hand to read the mind. $P s y$ chological Review, 100, 279-297.

HACKENBERG, T. D., \& JOKER, V. R. (1994). Instructional versus schedule control of humans' choices in situations of diminishing returns. Journal of the Experimental Analysis of Behavior, 62, 367-383.

Holman, J., Goetz, E. M., \& BaER, D. M. (1977). The training of creativity as an operant and an examination of its general characteristics. In B. C. Etzel, J. M. LeBlanc, \& D. M. Baer (Eds.), New developments in behavioral research: Theory, method and application (pp. 441-471). Hillsdale, NJ: Erlbaum.

JANOS, P. M., \& RoBinson, N. M. (1985). Psychosocial development in intellectually gifted children. In F. D. Horowitz \& M. O'Brien (Eds.), The gifted and talented: Developmental perspectives (pp. 149-196). Washington, DC: American Psychological Association.

Johnson, P. E., Duran, A. S., Hassebrock, F., Moller, J., Prietula, M., Feltovich, P. J., \& Swanson, D. B. (1981). Expertise and error in diagnostic reasoning. Cognitive Science, 5, 235-283.

JOYCE, J. H., \& ChaSE, P. N. (1990). Effect of response variability on the sensitivity of rule-governed behavior. Journal of the Experimental Analysis of Behavior, 54, 251-262.

KunN, D., \& PheLPS, E. (1982). The development of problem-solving strategies. In H. W. Reese (Ed.), Advances in child development and behavior (Vol. 17, pp. 2-44). New York: Academic Press.

Lesgold, A., Rubinson, H., Feltovich, P., Glaser, R., Klopfer, D., $\&$ WANG, Y. (1988). Expertise in a complex skill: Diagnosing x-ray pictures. In M. T. H. Chi, R. Glaser, \& M. J. Farr (Eds.), The nature of expertise (pp. 311-342). Hillsdale, $\mathrm{NJ}$ : Erlbaum.

Machado, A. (1994). Polymorphic response patterns under frequencydependent selection. Animal Learning \& Behavior, 22, 53-71.
MECHNER, F. (1958). Probability relations within response sequences under ratio reinforcement. Journal of the Experimental Analysis of Behavior, 1, 109-121.

MechNer, F. (1991). The revealed operant: A way to study the characteristics of individual occurrences of operant responses. A Behavior Monograph. Cambridge, MA: Cambridge Center for Behavioral Studies.

Mechner, F., Hyten, C., Field, D. P., \& Madden, G. J. (1997). Using revealed operants to study the structure and properties of human operant behavior. Psychological Record, 47, 45-68.

Millenson, J. R., \& HuRwitz, H. M. B. (1961). Some temporal and sequential properties of behavior during conditioning and extinction. Journal of the Experimental Analysis of Behavior, 4, 97-106.

Miller, G. A., \& FrICK, F. C. (1949). Statistical behavioristics and sequences of responses. Psychological Review, 56, 311-324.

NeURINGER, A. (1992). Choosing to vary and repeat. Psychological Science, 3, 83-91.

Neuringer, A. (1993). Reinforced variation and selection. Animal Learning \& Behavior, 21, 83-91.

PAGE, S., \& NEURINGER, A. (1985). Variability is an operant. Journal of Experimental Psychology: Animal Behavior Processes, 11, 429-452.

Pyror, E. W., HaAG, R., \& O'Reilly, J. (1969). The creative porpoise: Training for novel behavior. Journal of the Experimental Analysis of Behavior, 12, 653-661.

ROYCE, J. (1898). The psychology of invention. Psychological Review, $5,113-144$.

SCHAUBLE, L. (1990). Belief revision in children: The role of prior knowledge and strategies for generating evidence. Journal of Experimental Child Psychology, 49, 31-57.

SCHOENFELD, W. N. (1968). On the difference in resistance to extinction following regular and periodic reinforcement. Journal of the Experimental Analysis of Behavior, 2, 259-261.

Shimoff, E., Catania, A. C., \& Matthews, B. A. (1986). Human operant performance: Sensitivity and pseudosensitivity to contingencies. Journal of the Experimental Analysis of Behavior, 46, 149-157.

SIEGLER, R. S. (1995). How does change occur: A microgenetic study of number conservation. Cognitive Psychology, 28, 225-273.

SiEgler, R. S., \& Crowley, K. (1991). The microgenetic method: A direct means for studying cognitive development. American Psychologist, 46, 606-620.

Siegler, R. S., \& Jenkins, E. (1989). How children discover new strategies. Hillsdale, NJ: Erlbaum.

SKINNER, B. F. (1981). Selection by consequences. Science, 213, 501-504.

Staddon, J. E. R. (1993). Adaptive behavior and learning. Cambridge: Cambridge University Press.

Stokes, P. D. (1995a). Learned variability. Animal Learning \& Behavior, 23, 164-176.

STOKES, P. D. (1995b). Teaching variability in problem solving. In R. Kelder (Ed.), Theories of learning: Teaching for understanding and creativity (pp. 1-8). New Paltz, NY: State University of New York Press.

Stokes, P. D. (in press). Learned variability theory: Implications for creativity. Creativity Research Journal.

Stokes, P. D., \& Balsam, P. (1991). Effects of reinforcing preselected approximations on the topography of the rat's barpress. Journal of the Experimental Analysis of Behavior, 12, 349-373

Wallace, D. B., \& Gruber, H. E. (1989). Creative people at work. New York: Oxford University Press.

WEINER, H. (1964). Conditioning history and human fixed-interval performance. Journal of the Experimental Analysis of Behavior. 7. 383-385.

(Manuscript received August 1, 1997: revision accepted for publication September 22, 1998.) 